\title{
O impacto do uso da metformina no câncer colorretal: revisão integrativa
}

\author{
The impact of metformin use in colorectal cancer: an integrative review
}

\section{El impacto del uso de metformina en el cáncer colorrectal: una revisión integradora}

Camila Santana Silva ${ }^{1 *}$, Márcia Maria Aguiar de Jesus Carneiro¹, Giovanna Lima Freitas Flôres¹, Maria Luzia Santana Cabral ${ }^{1}$, Rúbens Costa Cardoso ${ }^{1}$, Brenda de Sá Reis ${ }^{1}$, Shesllen Mikaelly Cruz Corrêa ${ }^{1}$, Maíra Caroline Silva Rodrigues², Gustavo de Carvalho Ruas ${ }^{1}$, Fillipe Dantas Pinheiro'.

\section{RESUMO}

Objetivo: Avaliar o impacto do uso da metformina como droga antioncogênica no câncer colorretal. Métodos: Este trabalho constitui de uma revisão integrativa baseada na seleção criteriosa de artigos com a temática do impacto da metformina no câncer colorretal publicados nos últimos 10 anos nas bases de dados Pubmed e BVS com a combinação de descritores "metformin", "colorectal cancer", "colon cancer" e "rectal cancer". Resultados: Foram encontrados, analisados e descritos 6 artigos (100\%) sobre o referido tema, sendo que 4 (67\%) foram estudos clínicos randomizados e 2 (33\%) estudos clínicos controlados. Foi verificado que metformina pode impactar no câncer colorretal de duas formas distintas, porém não excludentes, atuando como uma quimioterapia, principalmente quimioterapia adjuvante à outras quimioterapias em pacientes com câncer de colorretal ou como quimioprevenção do câncer colorretal. Considerações finais: Os achados encontrados demonstram que apesar de poucas divergências a metformina pode ter um papel importante como droga antioncogênica atuando tanto na prevenção como no tratamento adjuvante do câncer colorretal, porém os estudos são iniciais e poucos, necessitando de maiores estudos clínicos para maior confiabilidade e utilização na prática clínica.

Palavras-chave: Câncer colorretal, Metformina, Prevenção, Tratamento.

\section{ABSTRACT}

Objective: To evaluate the impact of using metformin as an antioncogenic drug in colorectal cancer. Methods: This work is an integrative review based on the careful selection of articles on the impact of metformin on colorectal cancer published in the last 10 years in Pubmed and BVS databases with the combination of descriptors "metformin", "colorectal cancer", "colon cancer" and "rectal cancer". Results: Six articles (100\%) on the subject were found, analyzed and described, with $4(67 \%)$ being randomized clinical studies and $2(33 \%)$ controlled clinical studies. It was found that metformin can impact colorectal cancer in two distinct but not exclusive ways, acting as a chemotherapy, mainly adjuvant chemotherapy to other chemotherapy in patients with colorectal cancer or as a chemoprevention of colorectal cancer. Final considerations: The findings show that, despite few divergences, metformin can play an important role as an antioncogenic drug acting both in the prevention and in the adjuvant treatment of colorectal cancer, but the studies are initial and few, requiring further clinical studies for greater reliability and use in clinical practice.

Keywords: Colorectal cancer, Metformin, Prevention, Treatment.

\section{RESUMEN}

Objetivo: Evaluar el impacto del uso de metformina como fármaco antioncogénico en el cáncer colorrectal. Métodos: Este trabajo es una revisión integradora basada en la cuidadosa selección de artículos sobre el impacto de la metformina en el cáncer colorrectal publicados en los últimos 10 años en las bases de datos Pubmed y BVS con la combinación de descriptores "metformina", "cáncer colorrectal", "cáncer de colon "y" cáncer de recto ". Resultados: Se encontraron, analizaron y describieron seis artículos (100\%) sobre el tema, siendo 4 (67\%) estudios clínicos aleatorizados y 2 (33\%) estudios clínicos controlados. Se encontró que la metformina puede afectar el cáncer colorrectal de dos formas distintas, pero no exclusivas, actuando como quimioterapia, principalmente quimioterapia adyuvante a otra quimioterapia en pacientes con cáncer colorrectal o como quimioprevención del cáncer colorrectal. Consideraciones finales: Los hallazgos muestran que, a pesar de pocas divergencias, la metformina puede jugar un papel importante como fármaco antioncogénico actuando tanto en la prevención como en el tratamiento adyuvante del cáncer colorrectal, pero los estudios son iniciales y escasos, requiriendo más estudios clínicos para mayor fiabilidad y uso en la práctica clínica.

Palabras clave: Cáncer colorrectal, Metformina, Prevención, Tratamiento.

\footnotetext{
${ }^{1}$ Faculdade Santo Agostinho (FASA), Vitória da Conquista - BA. *E-mail: camila_santana01@hotmail.com
}

2 Faculdade Santo Agostinho (FASA), Itabuna - BA.

SUBMETIDO EM: 8/2021

ACEITO EM: 8/2021

PUBLICADO EM: 9/2021 


\section{INTRODUÇÃO}

Atualmente o carcinoma colorretal (CCR) é considerado como a terceira neoplasia mais comum em homens e a segunda mais comum em mulheres no mundo. No Brasil, o CCR é considerado o terceiro câncer mais frequente, sendo confirmados cerca de 36 mil novos casos no ano de 2018 (CAMPOS FG, et al., 2017). Apesar dos avanços em diagnóstico e tratamento, a mortalidade ainda se mantém alta nesse tipo de câncer e praticamente estagnada nos últimos 40 anos revelando uma sobrevida média global em cinco anos em torno de $55 \%$ nos países desenvolvidos e $40 \%$ em países em desenvolvimento (MOURA SF, et al., 2020).

No momento do diagnóstico, $40 \%$ dos pacientes apresentam um tumor localizado do intestino grosso, $26 \%$ apresentam metástases nos linfonodos regionais e quase $20 \%$ apresentam metástases à distância (SIEGEL $\mathrm{RL}$, et al., 2019). O câncer colorretal surge a partir de alterações genéticas de células da mucosa colônica normal que evoluem para adenomas e adenocarcinomas e atualmente existem múltiplas modalidades terapêuticas para tratamento, desde cirurgia (curativa ou paliativa), a quimioterapia e radioterapia, nesse caso em especial para câncer de reto, podendo ser utilizadas em associação ou não (SILVA M e ERRANTE PR, 2017

Além disso, a maior parcela da população acometida por esses tumores está em idade avançada (média de diagnóstico é superior a 50 anos) e normalmente apresenta várias doenças concomitantes. Uma dessas doenças é o diabetes mellitus (DM) tipo 2, com uma incidência cada vez maior, é caracterizada por deficiência no metabolismo da glicose e uma das principais causas de morte no ocidente. Está associado a várias complicações e a um risco aumentado de desenvolver tumores sólidos (HENRICH GR e CALVETTI PÜ, 2019). Vários estudos demonstraram a relação entre diabetes e câncer e suas associações significativas com câncer colorretal, próstata, pâncreas, endométrio e mama (ANWAR MA, et al., 2014).

Por conta dessa ligação entre o DM tipo 2 e o câncer colorretal outros estudos foram desenvolvidos e demonstraram a existência de mais de 200 agentes com efeitos protetores contra o desenvolvimento do CCR, entre eles a metformina (SEHDEV A e O'NEIL BH, 2015).

A metformina é um medicamento anti-hiperglicêmico, da classe biguanida, mais usado como terapia farmacológica em pacientes com diabetes tipo 2. Além de sua função como supressor da gliconeogênese, a metformina é também um sensibilizador à insulina que melhora a resistência à insulina e diminui os níveis plasmáticos da mesma em jejum (PASCHOAL C, et al., 2019). Recentemente demonstrou-se que a metformina possui fortes propriedades antioncogênica através de vários mecanismos moleculares (NANGIAMAKKER P, et al., 2014).

Diante disso, o presente constructo tem como objetivo avaliar qual o impacto do uso da metformina como droga antioncogênica no câncer colorretal.

\section{MÉTODOS}

Este artigo trata-se de um estudo teórico, através de uma pesquisa qualitativa e descritiva com coletas realizadas a partir de fontes secundárias, por meio de levantamento bibliográfico configurando uma revisão integrativa da literatura. Dessa forma, seguindo a primeira etapa de uma revisão sistemática, foi elaborada a seguinte questão de pesquisa: Qual o impacto do uso da metformina como droga antioncogênica no câncer colorretal?

Foi realizada pesquisa nos bancos de dados eletrônicos PubMed, Scientific Electronic Libray Online (SciELO), Literatura Latino-Americana e do Caribe (Lilacs) e Biblioteca Virtual em Saúde (BVS) a fim de abarcar uma maior quantidade de estudos, porém não foram achados artigos acerca do tema nas bases SciELO e Lilacs, ficando apenas com artigos encontrados no PubMed e BVS. A busca foi efetuada no período de setembro a novembro de 2020 seguindo as recomendações do protocolo Preferred Reporting Items for Systematic Reviews and Meta-Analyses, a fim de promover transparência sobre métodos e procedimentos utilizados (Figura 1) (OKOLI C, et al., 2019, CAMARGO FC, et al., 2017).

As seguintes palavras-chaves foram empregadas, através do uso de booleanos, incluídos nos Descritores em Ciências da Saúde (DeCS), em suas variantes na língua inglesa: "Colorectal cancer" OR "Colon cancer" OR "Rectal cancer" AND "Metformin". 
Como critérios de inclusão tem-se estudos que estivessem disponíveis na íntegra, publicados no período de 2010 a 2020, com intuito de incluir estudos mais recentes, que tratavam estritamente do uso da metformina associada ao câncer colorretal em suas vertentes: atuação como prevenção ou como tratamento. Foram incluídos artigos de todos os idiomas e apenas artigos originais de estudos clínicos controlados e estudos clínicos randomizados de todas as fases por acreditar ter um maior impacto na prática clínica no ambiente da saúde seguindo a classificação de níveis de evidência em que estudos clínicos tem grau de recomendação $A$ e nível de evidencia 1B (FARIA L, et al., 2021).

Como critérios de exclusão têm-se artigos como revisões de literatura, relatos de caso, estudos observacionais de coorte e caso controle, estudos não experimentais e outras literaturas que não fossem artigos. Não foram considerados artigos fora do período selecionado e nem que se distanciavam do objetivo da pesquisa. Após a aplicação dos critérios de inclusão e exclusão, foram selecionados 92 artigos para revisão. Os artigos foram agrupados em duas categorias temáticas: 1) Metformina atuando na prevalência do câncer colorretal, 2) Metformina atuando na incidência do câncer colorretal.

Figura 1 - Fluxograma representativo da metodologia de inclusão dos artigos neste estudo.

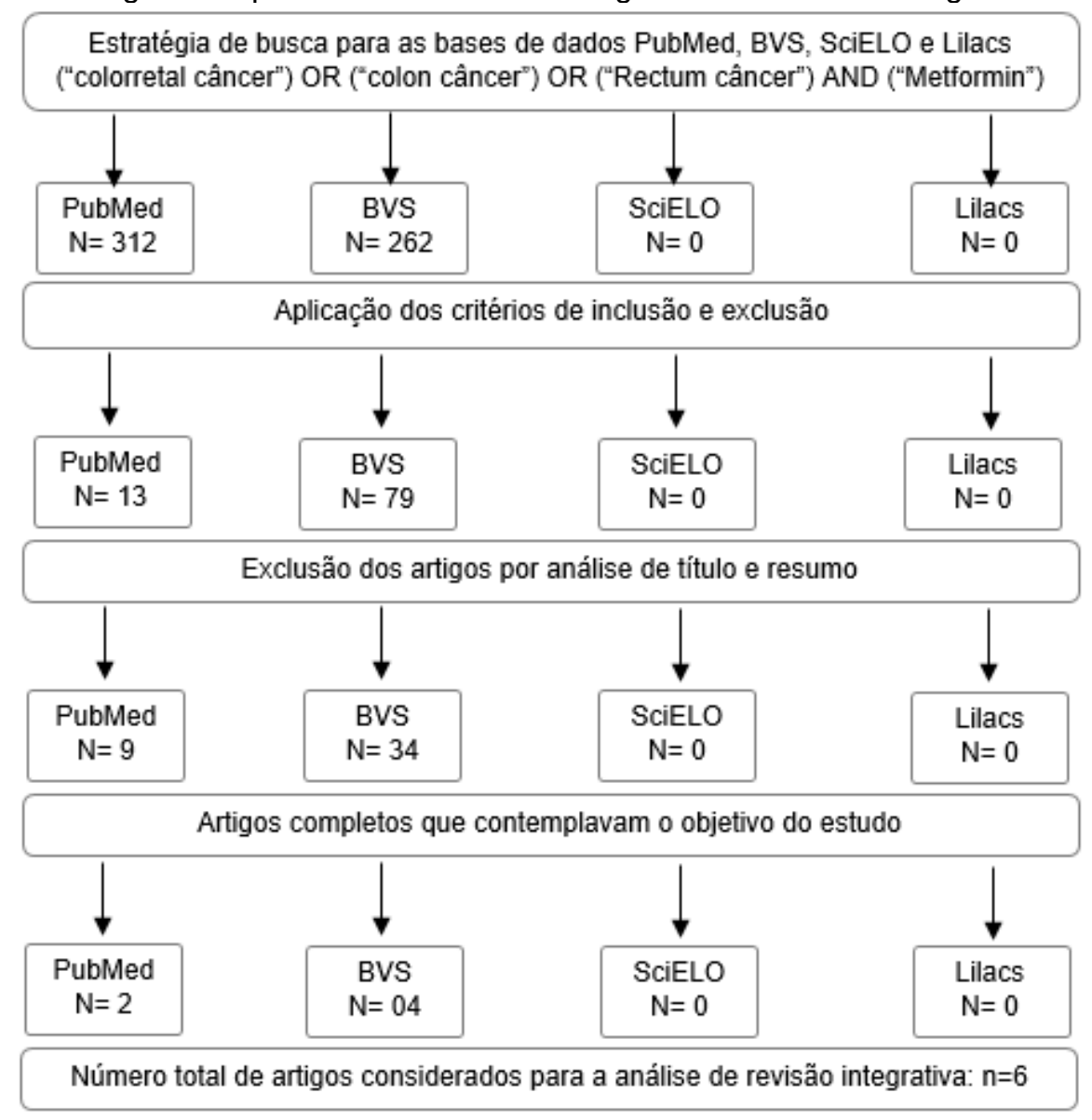

Fonte: Silva CS, et al., 2021.

\section{RESULTADOS}

Os artigos selecionados e analisados tratam-se de ensaios clínicos, contudo os artigos tiveram diferentes vertentes/categorias temáticas a serem analisadas pelo problema proposto. Dos 6 artigos (100\%) analisados, $2(33,3 \%)$ abordaram o uso da metformina como um quimioterápico capaz de ser utilizado no tratamento de pacientes que já estavam diagnosticados com câncer colorretal (CCR), verificando então a atuação da metformina na prevalência do CCR. Os outros 4 artigos (66,6\%) abordaram o uso da metformina como uma quimioprevenção capaz de ser utilizado como forma de evitar o desenvolvimento de câncer colorretal em pacientes que não possuíam o mesmo, avaliando no caso a atuação da metformina na incidência do CCR. 
Dentre os 2 artigos (100\%) que verificaram o uso da metformina na prevalência do câncer colorretal, 1 artigo (50\%) analisou a utilização da metformina de forma sozinha e combinada com exercícios em pacientes com CCR e o outro artigo (50\%) analisou a combinação da metformina com outros quimioterápicos em pacientes com CCR. Dentre os 4 artigos (100\%) que verificaram o uso da metformina na incidência do câncer colorretal, 2 artigos (50\%) analisaram o efeito da metformina em focos de criptas aberrantes e 2 artigos (50\%) analisaram o efeito da metformina em pólipos adenomatosos ou hiperplásicos e adenomas.

No que se refere à metodologia adotada, verificaram-se os seguintes delineamentos: 1) Ensaio clínico randomizado: quatro; 2) Ensaio clinico controlado: dois. No Quadro 1 são apresentados os artigos selecionados e analisados de acordo a cronologia decrescente das publicações, autor, título do artigo, país, revistas em que os mesmos foram publicados e fator de impacto da revista segundo o uso da metformina na prevalência e na incidência do CCR, respectivamente.

Quadro 1 - Artigos selecionados de acordo com ano de publicação, autor, título, país, revista e fator de impacto da revista segundo o uso da metformina na prevalência do CCR.

\begin{tabular}{|c|c|c|c|c|}
\hline Autor/ano & Título & País & Revista & $\begin{array}{l}\text { Fator de } \\
\text { impacto }\end{array}$ \\
\hline $\begin{array}{l}\text { BROWN JC, et } \\
\text { al. (2020) }\end{array}$ & $\begin{array}{l}\text { Efeito do exercício ou da } \\
\text { metformina nos biomarcadores da } \\
\text { inflamação no câncer de mama e } \\
\text { colorretal: um ensaio randomizado }\end{array}$ & EUA & $\begin{array}{l}\text { Cancer } \\
\text { Prevention } \\
\text { Research }\end{array}$ & 8.590 \\
\hline $\begin{array}{l}\text { MIRANDA VC, } \\
\text { et al. (2016) }\end{array}$ & $\begin{array}{l}\text { Ensaio de fase } 2 \text { de metformina } \\
\text { combinada com } 5 \text {-fluorouracil em } \\
\text { pacientes com câncer colorretal } \\
\text { metastático refratário }\end{array}$ & BRASIL & $\begin{array}{l}\text { Clinical } \\
\text { Colorectal } \\
\text { Cancer }\end{array}$ & 3.120 \\
\hline $\begin{array}{l}\text { ZELL JÁ, et } \\
\text { al.(2020) }\end{array}$ & $\begin{array}{l}\text { Um ensaio de fase lla de } \\
\text { metformina para redução do risco } \\
\text { de câncer colorretal entre indivíduos } \\
\text { com história de adenomas } \\
\text { colorretais e índice de massa } \\
\text { corporal elevado. }\end{array}$ & EUA & $\begin{array}{l}\text { Cancer } \\
\text { Prevention } \\
\text { Research }\end{array}$ & 8.590 \\
\hline $\begin{array}{l}\text { HIGURASHI T, } \\
\text { et al. (2016) }\end{array}$ & $\begin{array}{l}\text { Metformina para quimioprevenção } \\
\text { de adenoma colorretal metacrônico } \\
\text { ou pólipos em pacientes pós- } \\
\text { polipectomia sem diabetes: um } \\
\text { estudo multicêntrico duplo-cego, } \\
\text { controlado por placebo, } \\
\text { randomizado de fase } 3\end{array}$ & JAPÃO & $\begin{array}{l}\text { The Lancet } \\
\text { Oncology }\end{array}$ & 34.340 \\
\hline $\begin{array}{l}\text { ZHAO X, et al. } \\
\qquad(2015)\end{array}$ & $\begin{array}{l}\text { Efeitos de diferentes doses de } \\
\text { tratamento com metformina por } 6 \\
\text { meses em focos de cripta aberrante } \\
\text { em pacientes chineses com } \\
\text { tolerância à glicose diminuída }\end{array}$ & CHINA & $\begin{array}{l}\text { European } \\
\text { Journal of } \\
\text { Cancer } \\
\text { Prevention }\end{array}$ & 2.310 \\
\hline $\begin{array}{l}\text { HOSONO K, et } \\
\text { al.(2010) }\end{array}$ & $\begin{array}{l}\text { Metformina suprime focos de cripta } \\
\text { aberrante colorretal em um ensaio } \\
\text { clínico de curto prazo }\end{array}$ & JAPÃO & $\begin{array}{l}\text { Cancer } \\
\text { Prevention } \\
\text { Research }\end{array}$ & 8.590 \\
\hline
\end{tabular}

Fonte: Silva CS, et al., 2021.

Nos Quadros 2 e 3 são apresentados os artigos segundo os objetivos, metodologia empregada com destaque para a duração no ensaio, o tamanho da amostra e a dosagem da metformina utilizada, as limitações dos estudos e os resultados segundo o uso da metformina na prevalência e na incidência do CCR, respectivamente. 


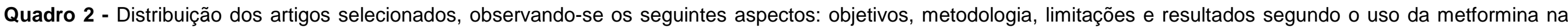
prevalência do CCR.

\begin{tabular}{|c|c|c|c|c|}
\hline Autor/ano & Objetivo & Método & Limitações & Resultados \\
\hline $\begin{array}{l}\text { BROWN JC, et al. } \\
\qquad(2020)\end{array}$ & $\begin{array}{l}\text { Testar o efeito do exercício e da } \\
\text { metformina em medidas de resultado } \\
\text { de inflamação pré-especificadas em } \\
\text { pacientes com câncer de mama e } \\
\text { colorretal. }\end{array}$ & $\begin{array}{l}\text { Ensaio clinico de fase II, } \\
\text { multicêntrico, randomizado, quatro } \\
\text { braços. Duração: } 12 \text { semanas. } \\
\text { Amostra: } 139 \quad \text { pacientes. } \\
\text { Metformina: } 1700 \mathrm{mg} / \text { dia. }\end{array}$ & $\begin{array}{l}\text { Pequeno tamanho de } \\
\text { amostra e pequena } \\
\text { duração de intervenção. }\end{array}$ & $\begin{array}{l}\text { Em pacientes com câncer de mama e colorretal com baixa } \\
\text { atividade física inicial e sem diabetes tipo } 2 \text { o exercício e a } \\
\text { metformina inibem processos inflamatórios distintos, e a } \\
\text { combinação de exercícios e metformina inibem de forma } \\
\text { abrangente. }\end{array}$ \\
\hline $\begin{array}{l}\text { MIRANDA VC, et } \\
\text { al. } \\
\text { (2016) }\end{array}$ & $\begin{array}{l}\text { Avaliar a eficácia e segurança da } \\
\text { metformina combinada com } \\
\text { fluorouracil (5-FU) em pacientes com } \\
\text { câncer colorretal refratária e } \\
\text { progressiva. }\end{array}$ & $\begin{array}{l}\text { Ensaio clínico de fase II, centro } \\
\text { único e um braço, não randomizado. } \\
\text { Duração: } 8 \text { semanas. Amostra: } 50 \\
\text { pacientes. Metformina: } 850 \text { mg mais } \\
5 \text {-FU } 425 \mathrm{mg} / \mathrm{m}^{2} \text { e leucovorina } 50 \\
\text { mg. }\end{array}$ & $\begin{array}{lr}\text { Ensaio de } & \text { centro } \\
\text { pacientesico, } \\
\text { refratários, } & \text { não tão } \\
\text { amostra. } & \text { pequena }\end{array}$ & $\begin{array}{l}22 \% \text { dos pacientes apresentaram estabilização do tumor } \\
\text { após } 8 \text { semanas. A utilização conjunta das substâncias teve } \\
\text { uma média geral de sobrevida livre de progressão (PFS) de } \\
2 \text { meses e uma média de sobrevida global (OS) de } 7,9 \\
\text { meses, mas entre os pacientes que experimentaram o } \\
\text { controle da doença a estabilização da doença teve uma PFS } \\
\text { e OS maior. }\end{array}$ \\
\hline
\end{tabular}

Fonte: Silva CS, et al., 2021.

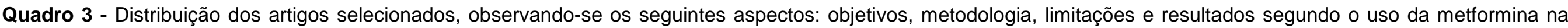
incidência do CCR.

\begin{tabular}{|c|c|c|c|c|}
\hline Autor/ano & Objetivo & Método & Limitações & Resultados \\
\hline $\begin{array}{l}\text { ZELL JÁ, et al. } \\
\qquad(2020)\end{array}$ & $\begin{array}{l}\text { Testar se a metformina oral afeta } \\
\text { níveis de Proteína Ribossômica Fosfo- } \\
\text { S6 Ser235 no tecido retal entre } \\
\text { pacientes obesos com adenoma } \\
\text { colorretal. }\end{array}$ & $\begin{array}{l}\text { Ensaio clínico de fase } \text { II, } \\
\text { multicêntrico e um braço, não } \\
\text { randomizado. Duração: } 12 \\
\text { semanas. Amostra: } 32 \text { pacientes. } \\
\text { Metformina: } 2000 \mathrm{mg} / \text { dia. }\end{array}$ & Não foi especificado. & $\begin{array}{l}\text { Não houve diferenças no tecido retal após } 12 \text { semanas de } \\
\text { tratamento com metformina entre pacientes obesos com } \\
\text { adenoma colorretal. }\end{array}$ \\
\hline $\begin{array}{l}\text { HIGURASHI T, et } \\
\text { al. } \\
\text { (2016) }\end{array}$ & $\begin{array}{l}\text { Avaliar a segurança e o efeito } \\
\text { quimiopreventivo da metformina em } \\
\text { câncer colorretal (CCR) esporádico } \\
\text { (avaliado por adenoma e recorrência } \\
\text { de pólipo) em pacientes com alto risco } \\
\text { de recorrência de adenoma. }\end{array}$ & 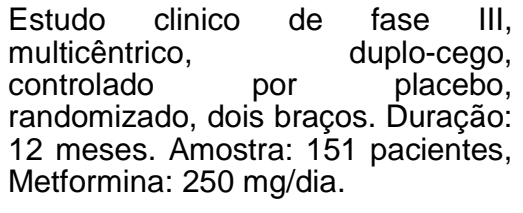 & $\begin{array}{l}\text { Não foi feito estudo de } \\
\text { dose-resposta do efeito da } \\
\text { metformina, pequena } \\
\text { amostra e curto período da } \\
\text { intervenção. }\end{array}$ & $\begin{array}{l}\text { A prevalência de pólipos totais e de adenomas no grupo } \\
\text { metformina foi significativamente menor do que no grupo } \\
\text { placebo. }\end{array}$ \\
\hline $\begin{array}{l}\text { ZHAO X, et al. } \\
\qquad(2014)\end{array}$ & $\begin{array}{l}\text { Avaliar os efeitos quimiopreventivos } \\
\text { de diferentes doses de metformina por } \\
6 \text { meses em focos de cripta aberrante } \\
\text { retal (ACF) em pacientes com } \\
\text { tolerância à glicose diminuída (IGT). }\end{array}$ & $\begin{array}{l}\text { Ensaio clinico randomizado, centro } \\
\text { único, aberto e controlado em } \\
\text { paralelo, de quatro braços. Duração: } \\
6 \text { meses. Amostra: } 120 \text { pacientes. } \\
\text { Metformina: doses de } 250 \mathrm{mg} / \text { dia, } \\
500 \mathrm{mg} / \text { dia e } 1500 \mathrm{mg} / \mathrm{dia} \text {. }\end{array}$ & $\begin{array}{l}\text { Relativamente pequena } \\
\text { amostra, ser centro único, } \\
\text { curta duração e não ser um } \\
\text { ensaio duplo- cego. }\end{array}$ & $\begin{array}{l}\text { A metformina em } 500 \text { e } 1500 \mathrm{mg} / \mathrm{dia} \text {, mas não em } 250 \mathrm{mg} / \\
\text { dia, por } 6 \text { meses, efetivamente suprimiu a formação de ACF } \\
\text { em pacientes com IGT. }\end{array}$ \\
\hline $\begin{array}{l}\text { HOSONO K, et al. } \\
(2010)\end{array}$ & $\begin{array}{l}\text { Avaliar o efeito quimiopreventivo da } \\
\text { metformina sobre focos de cripta } \\
\text { aberrante retal (ACF), bem como } \\
\text { atividade proliferativa no epitélio } \\
\text { colônico e atividade apoptótica. }\end{array}$ & $\begin{array}{l}\text { Ensaio clinico controlado, centro } \\
\text { único, randomizado, dois braços. } \\
\text { Duração: } 1 \text { mês. Amostra: } 26 \\
\text { pacientes. Metformina: } 250 \mathrm{mg} / \mathrm{dia} \text {. }\end{array}$ & $\begin{array}{l}\text { Pequena amostra e curta } \\
\text { duração. }\end{array}$ & $\begin{array}{l}\text { Em } 1 \text { mês, o grupo metformina teve uma diminuição no no } \\
\text { médio de ACF por paciente em relação ao grupo controle. A } \\
\text { atividade proliferativa no epitélio colônico foi reduzido e } \\
\text { atividade apoptótica permaneceu igual. }\end{array}$ \\
\hline
\end{tabular}

Fonte: Silva CS, et al., 2021. 


\section{DISCUSSÃO}

De acordo com os critérios adotados, foram encontrados um número reduzido de estudos, o que indica escassez na literatura de pesquisas que abordam a temática. Por conta dessa situação, mesmo apresentando delineamento e objetivos específicos diferentes, a análise foi feita partindo do objetivo macro dos estudos que entram em consonância com o problema de pesquisa que este artigo deseja responder.

\section{Metformina atuando na prevalência do câncer colorretal}

Desde que dados observacionais iniciais foram publicados sobre as propriedades antioncogênicas da metformina, vários estudos in vitro e in vivo foram desenvolvidos para entender o mecanismo molecular antitumorigênico que a metformina atua, descobrindo então atuações de diversas formas desde a ativação da 5'-monofosfato-adenosina proteína quinase ativada (AMP-Q), que por sua vez inibe o alvo da rapamicina em mamíferos (mTOR), sendo a maioria através desse, até vias de proteção não dependentes da AMPK que incluem redução do fator de crescimento semelhante à insulina 1, leptina, bloqueio da sinalização de HER-2, inibição da angiogênese, potencialização da adiponectina e através das vias inflamatórias (MALLIK $R$ e CHOWDHURY TA, 2018; ZHAO J, 2016).

Segundo Brown JC, et al. (2020) traz como proposta a avaliação do exercício e o uso da metformina na atuação de determinados processos inflamatórios específicos em pacientes com câncer de mama e colorretal estágio I- III. Os resultados mostraram que em comparação com o grupo controle, o exercício sozinho reduziu hs-CRP e IL-6, mas não alterou o sTNF- $\alpha$ R2, a metformina sozinha não alterou significativamente nenhuma das medidas de inflamação, porém os dois em conjunto, exercício e metformina, reduziram significativamente sTNF-aR2 e IL-6, mas não alterou significativamente a hs-CRP.

Os resultados desse estudo podem ser associados com os dados de Cruzs SM e Balkwill FR (2015); Figueiredo CRLV (2019) e Johnson DE, et al. (2018) que demonstram que mediadores do sistema imunológico inato são detectados na maioria dos cânceres independentemente se a inflamação está ou não implicada no seu desenvolvimento, sendo assim células tumorais podem regular a expressão de citocinas, quimiocinas e outros mediadores através de vias de sinalização para favorecer o recrutamento de células imunes circulantes e promover a sobrevivência, proliferação, migração e invasão neoplásica.

Entre elas estão a via JAK/STAT que é ativada pela IL-6 e a via do NF-kB que é ativada pelo TNF- $\alpha$ por meio do seu receptor, sTNF-aR2. Assim se a metformina está implicada na diminuição desses mediadores inflamatórios (IL- 6 e TNf-alfa) isso significa que pode contribuir indiretamente para a diminuição de alterações neoplásicas nas células (CRUSZ SM e BALKWILL FR, 2015).

Além da ação nos mediadores inflamatórios, a metformina foi analisada em ação como quimioterapia adjuvante a outros quimioterápicos como é o caso do estudo realizado por Miranda VC, et al. (2016) que avaliou a eficácia e segurança do uso da metformina aliada ao 5 -fluorouracil (5-FU) em pacientes com câncer colorretal refratário (mCRC) e progressivo (KOBIELA J, et al., 2019).

O resultado deste ensaio trouxe que entre 50 pacientes incluídos, $11(22 \%)$ estabilizaram o tumor em 8 semanas demonstrando que a utilização conjunta das substâncias tem uma média geral de sobrevida livre de progressão (PFS) de 2 meses e uma média de sobrevida global (OS) de 7,9 meses mas entre os pacientes que experimentaram o controle da doença, a estabilização da doença teve um aumento de $2,8 \%$ no PFS e de $2,5 \%$ no OS levando à conclusão de que é possível a metformina ter uma efeito antitumoral em pacientes com câncer colorretal refratário mesmo sem poder descartar possíveis benefícios secundários a biologia do tumor, reexposição ao 5-FU ou o por acaso.

Além disso, apresenta a hipótese de que o efeito antitumoral da metformina nos pacientes com câncer colorretal em especial refratários no ensaio clinico pode partir do pressuposto que a metformina inibiu o metabolismo de células tronco cancerígenas, conhecidas por serem quimiorresistentes e por isso estarem presentes nos cânceres refratários (ZHAO J, 2016). Essa hipótese vem do embasamento descrito por Yang JÁ, et al. (2016) e Czeczko LEA, et al. (2020) que demonstraram que a metformina apresenta efeitos citotóxicos em células tronco cancerígenas inibindo a via inflamatória (principalmente a da NF-kB) e a via glicolítica necessária para formação e transformação das células tronco cancerígenas. 
Dessa forma o estudo de Brown JC, et al. (2020) e o de Miranda VC, et al. (2016) apresentam concordância quanto a ação da metformina nas vias inflamatórias e sua ação no microambiente tumoral. Os dois principais estudos clínicos trazem dados relevantes para considerar o impacto da metformina no prognóstico de pacientes com câncer colorretal, atuando na prevalência da doença seja em conjunto à um quimioterápico ou sozinha. Contudo as informações ainda são primárias e divergentes necessitando de estudos mais profundos e criteriosos.

\section{Metformina atuando na incidência do câncer colorretal}

Segundo Melo IJR, et al. (2019) e Piazza T, et al. (2021) a maioria dos tumores colorretais origina-se de pólipos sejam adenomas ou pólipos hiperplásicos, que de início são neoplasias benignas situadas no cólon e/ou reto mas podem sofrer malignização com tempo, sendo considerados alto fatores de risco para 0 desenvolvimento de pólipos colorretal metacrônicos, câncer colorretal ou ambos. Existe também os Focos de Criptas Aberrantes (ACF) formados por criptas aberrantes identificadas como lesões pré- neoplásicas devido à presença de displasias teciduais, que também são fatores de risco para o desenvolvimento de câncer colorretal (FIGUEIREDO CRLV, 2019).

Seguindo essa linha de raciocínio, foi realizado um estudo clinico no Japão por Higurashi T, et al. (2016) com o objetivo de avaliar a segurança e os efeitos quimiopreventivos da metformina no câncer colorretal esporádico (avaliado por adenoma e recorrência de pólipo) em pacientes com alto risco de recorrência de adenoma. Os resultados demonstraram que a prevalência de pólipos tanto hiperplásicos e adenomatosos como adenomas foi significativamente menor no grupo que usava metformina do que no grupo placebo, concluindo que a metformina tem um papel importante na quimioprevenção do câncer colorretal.

Este ensaio contrasta com os resultados encontrados no ensaio de clinico de Zell JÁ, et al. (2020) que mostrou que após 12 semanas de tratamento não houve diferenças no tecido retal normal dos pacientes significando que a metformina através da via mTOR não suprime a proliferação celular nem a via apoptótica.

Existem diferenças entre os estudos de Zell JÁ, et al. (2020) e Higurashi T, et al. (2016) que devem ser levados em conta para explicar os resultados divergentes. O estudo de Zell JÁ, et al. (2020) foi um estudo de fase 2 com todos os pacientes obesos (IMC>30), com uma duração menor, de apenas 12 semanas, com altas doses de metformina ( $2000 \mathrm{mg} / \mathrm{dia}$ ) e consequente altos efeitos colaterais, concentrando em um desfecho de biomarcadores e com foco em avaliar o tecido alvo de origem (mucosa retal normal). Já o estudo de Higurashi T, et al. (2016) foi um estudo de fase $3 \mathrm{com}$ pacientes com média de IMC de $23 \mathrm{~kg} / \mathrm{m}^{2}$, com duração maior de 1 ano, com dose de metformina menor $(250 \mathrm{mg})$ obtendo menos efeitos adversos e comprovando melhor eficácia, concentrando mais em um desfecho clínico primário (recorrência de adenoma).

Apesar divergir do estudo de Zell JÁ, et al. (2020), o estudo de Higurashi T, et al. (2016) concorda em objetivo macro com outros dois estudos clínicos selecionados como os estudos de Hosono K, et al. (2010) e Zhao X, et al. (2015) que também trazem a análise da metformina como quimioprevenção, porém na atuação em focos de criptas aberrantes (ACF).

O estudo clinico de Hosono K, et al. (2010) avaliou o efeito quimiopreventivo da metformina sobre os ACF onde os resultados demonstraram que após um mês o grupo em tratamento com metformina teve uma diminuição significativa no número médio de ACF por paciente $(\mathrm{p}=0,007)$ enquanto o número médio de ACF não mudou significativamente no grupo controle $(p=0,609)$. $O$ índice de antígeno nuclear de proliferação celular foi significativamente reduzido no grupo da metformina, porém o índice de células apoptóticas manteve-se inalterado no grupo da metformina demonstrando que o mesmo possui atuação supressiva na proliferação e formação de ACF e nenhuma ação apoptótica nas mesmas.

Já o estudo de Zhao X, et al. (2015) avaliou também os efeitos quimiopreventivos da metformina, porém em diferentes doses em pacientes com ACF e tolerância a glicose diminuída (IGT) por 6 meses. Este foi um estudo clinico que demonstrou que em pacientes com IGT, o número médio de ACF por paciente diminuiu significativamente após intervenção com metformina por 6 meses nas doses de $500 \mathrm{mg} / \mathrm{dia}$ e $1500 \mathrm{mg} / \mathrm{dia}$, mas não na dose de $250 \mathrm{mg} / \mathrm{dia}$ contrastando com os resultados encontrados por Higurashi T, et al. (2016) que utilizou a dosagem de $250 \mathrm{mg} /$ para os pólipos adenomatosos e obteve resultado positivo e o estudo de Hosono K, et al. (2010) que também obteve resultado positivo com apenas $250 \mathrm{mg} / \mathrm{dia}$ de metformina. 
Porém os pacientes desses dois estudos não possuíam tolerância à glicose diminuída o que pode ser um fator a ser considerado nessa diferença. Mesmo com algumas divergências em metodologia e dosagem da metformina, os quatros estudos demonstram um impacto na quimioprevenção do câncer colorretal principalmente no que diz respeito em diminuir o número de pólipos, adenomas ou ACF, podendo talvez no futuro utilizar da metformina como parte de um protocolo de prevenção de câncer colorretal.

\section{CONSIDERAÇÕES FINAIS}

Os artigos analisados mostraram que a metformina pode impactar no câncer colorretal de duas formas distintas, porém não excludentes, atuando como uma quimioterapia, principalmente quimioterapia adjuvante à outras quimioterapias em pacientes com câncer de colorretal ou como quimioprevenção do câncer colorretal. Os artigos trazem algumas divergências quanto a dose necessária para suprimir focos de criptas aberrantes e quanto a ação do mesmo em biomarcadores de pólipos e adenomas, contudo a prevalência dos resultados demonstra que a metformina pode ter um papel importante no mundo da oncologia gastrointestinal, principalmente como um agente de prevenção já que os resultados dos artigos foram mais favoráveis a esta vertente.

\section{REFERÊNCIAS}

1. ANWAR MA, et al. Colorectal and prostate cancer risk in diabetes: metformin, an actor behind the scene. Journal of Cancer, 2014; 5(9): 736.

2. BROWN JC, et al. Effect of Exercise or Metformin on Biomarkers of Inflammation in Breast and Colorectal Cancer: A Randomized Trial. Cancer Prevention Research, 2020; 13: 1055-62.

3. CRUSZ SM, BALKWILL FR. Inflammation and cancer: advances and new agents. Nature reviews Clinical oncology, 2015; $12(10):$ 584-596.

4. CAMARGO FC, et al. Modelos para a implementação da prática baseada em evidências na enfermagem hospitalar: revisão narrativa1. Texto Contexto Enfermagem, 2017; 26(4): e2070017.

5. CZECZKO LEA, et al. A expressão de marcadores de células-tronco e a análise do cd133 são relevantes na diferenciação do câncer colorretal?. ABCD. Arquivos Brasileiros de Cirurgia Digestiva, 2020; 33(4): e1568.

6. CAMPOS FG, et al. Incidência de câncer colorretal em pacientes jovens. Revista do Colégio Brasileiro de Cirurgiões, 2017; 44(2): 208-215.

7. FIGUEIREDO CRLV. O intrigante paradoxo da inflamação associada ao câncer: uma atualização. Jornal brasileiro de patologia e medicina laboratorial, 2019; v. 55: 321-332.

8. FARIA L, et. al. Medicina baseada em evidências: breve aporte histórico sobre marcos conceituais e objetivos práticos do cuidado. História, Ciências, Saúde-Manguinhos, 2021; 28: 59-78.

9. HENRICH GR, CALVETTI PÜ. Intervenção cognitiva e psicoeducativa em pacientes por câncer colorretal em ensaios clínicos randomizados: revisão sistemática. Revista da SBPH, 2019; 22(2): 211-227.

10. HIGURASHI T, et al. Metformin for chemoprevention of metachronous colorectal adenoma or polyps in postpolypectomy patients without diabetes: a multicentre double-blind, placebo-controlled, randomised phase 3 trial. The Lancet Oncology, 2016; 17(4): 475-483.

11. HOSONO K, et al. Metformin suppresses colorectal aberrant crypt foci in a short-term clinical trial. Cancer prevention research, 2010; 3(9): 1077-83.

12. JOHNSON DE, et al. Targeting the IL-6/JAK/STAT3 signalling axis in cancer. Nature Reviews Clinical Oncology, 2018; 12(4): 234.

13. KOBIELA J, et al. Metformin and Colorectal Cancer-A Systematic Review. Experimental and Clinical Endocrinology \& Diabetes, 2019; 127(7): 445-454.

14. MELO IJR, et al. Colonoscopia: Prevenção do Câncer Colorretal. Revista Científica Hospital Santa Izabel, 2019; 3(4): 218-225.

15. MALLIK R, CHOWDHURY TA. Metformin in cancer. Diabetes research and clinical practice, 2018; 143: 409-419.

16. MIRANDA VC, et al. Phase 2 trial of metformin combined with 5-fluorouracil in patients with refractory metastatic colorectal cancer. Clinical Colorectal Cancer, 2016; 15(4): 321-328.

17. MOURA SF, et al. Padrão Sintomatológico em Pacientes do Câncer Colorretal de acordo com a Idade. Revista Brasileira de Cancerologia, 2020; 66(1): e15474.

18. NANGIA-MAKKER P, et al. Metformin: a potential therapeutic agent for recurrent colon cancer. PloS one, 2014; 9(1): e84369.

19. OKOLI C, et al. Guia para realizar uma Revisão Sistemática de Literatura. EAD em Foco, 2019; 9(1): e748. 
20. PASCHOAL C, et al. Atividade antitumoral da metformina: uma nova aplicação para uma droga antiga. Perspectivas Médicas, 2019; 30(2): 33-39.

21. PIAZZA T, et al. Avaliação de diretrizes clínicas brasileiras em oncologia: carências no rigor do desenvolvimento, aplicabilidade e independência editorial. Cadernos de Saúde Pública, 2021; 37(4): e00031920.

22. SIEGEL RL, et al. Cancer statistics, 2019. CA a cancer journal for clinicians, 2019; 69(1): 7-34.

23. SEHDEV A, O'NEIL BH. The Role of Aspirin, Vitamin D, Exercise, Diet, Statins, and Metformin in the Prevention and Treatment of Colorectal Cancer. Curr Treat Options Oncol, 2015; 16: 43.

24. SILVA M, ERRANTE PR. Câncer colorretal: Fatores de risco, diagnóstico e tratamento. UNILUS Ensino e Pesquisa, 2017; 13 (33): 133-140.

25. YANG M, et al. Cancer stem cells, metabolism, and therapeutic significance. Tumor Biology, 2016; 37(5): 5735-5742.

26. ZELL JA, et al. A phase lla trial of metformin for colorectal cancer risk reduction among individuals with history of colorectal adenomas and elevated body mass index. Cancer Prevention Research, 2020; 13(2): 203-212.

27. ZHAO J. Cancer stem cells and chemoresistance: The smartest survives the raid. Pharmacology \& therapeutics, 2016 ; 160: 145-158.

28. ZHAO X, et al. Effects of different doses of metformin treatment for 6 months on aberrant crypt foci in Chinese patients with impaired glucose tolerance. European Journal of Cancer Prevention, 2015; 24(1): 27-36. 